SLAC-PUB-11463

BABAR-PROC-05/35

August 19, 2019

\title{
Electro-Weak Penguin and Leptonic Decays in BABAR
}

\author{
F. Di Lodovico \\ Queen Mary, University of London \\ Physics Department, Mile End Road, London E1 4NS, UK \\ (for the BABAR Collaboration)
}

\begin{abstract}
Electro-weak penguin and leptonic decays provide an indirect probe for physics beyond the Standard Model and contribute to the determination of Standard Model parameters. Copious quantities of $B$ mesons produced at the $B$-Factories permit precision measurements of the electro-weak penguin decays and searches for leptonic decays. We review the current experimental status of $b \rightarrow s(d) \gamma, B^{0} \rightarrow D^{* 0} \gamma, b \rightarrow s \ell^{+} \ell^{-}$and finally $B^{+} \rightarrow \tau^{+} \nu_{\tau}$ decays at BABAR.
\end{abstract}

Contributed to the Proceedings of the

10th International Conference on B-Physics at Hadron Machines, BEAUTY 2005,

6/20/2005-6/24/2005, Assisi (Perugia), Italy

Stanford Linear Accelerator Center, Stanford University, Stanford, CA 94309

Work supported in part by Department of Energy contract DE-AC02-76SF00515. 


\section{Introduction}

Electro-weak penguin decays are flavor-changing neutral current transitions that are forbidden in the Standard Model (SM) at tree level, but occur at loop level. Additional contributions to the electro-weak penguin loops could arise from New Physics effects such as new gauge bosons, charged Higgs bosons or supersymmetric particles. Study of electro-weak penguin decays constitutes an indirect probe for New Physics. In addition, these decays are relevant to the determination of the CKM matrix elements $\left|V_{u b}\right|$ and $\left|V_{t d} / V_{t s}\right|$ and to the measurement of the photon polarization.

In the SM, leptonic decays proceed via quark annihilation into a $W^{+}$boson: $\bar{b} u \rightarrow W^{+} \rightarrow \ell^{+} \nu_{\ell}$. The study of the purely leptonic $B^{+} \rightarrow \ell^{+} \nu_{\ell}$ can act as an indirect probe for New Physics and can provide sensitivity to poorly constrained SM parameters: $f_{B}$, which is the $B$ decay constant, and $\left|V_{u b}\right|$, which is the relevant CKM matrix element.

In this paper, recent results from $b \rightarrow s(d) \gamma, B^{0} \rightarrow D^{* 0} \gamma, b \rightarrow s \ell^{+} \ell^{-}$and $B^{+} \rightarrow \tau^{+} \nu_{\tau}$ decays at $B A B A R$ [1] are presented. The data sample used in the analyses ranges between 88 and 232 millions of $B \bar{B}$ events collected at the $\Upsilon(4 S)$ peak.

\section{$2 \quad b \rightarrow s \gamma$ final states}

Radiative decays involving the flavor-changing neutral current transition $b \rightarrow s$ are described in the SM primarily by a one-loop radiative penguin diagram containing a top quark and a $W$ boson. Additional contributions to the loop from New Physics, e.g. a charged Higgs boson or supersymmetric particles, could change the $b \rightarrow s \gamma$ rate [2, 3, 4, 5, 6, the direct $C P$ asymmetry between $b \rightarrow s \gamma$ and $\bar{b} \rightarrow \bar{s} \gamma$ decays [7], the direct asymmetry of the sum of $b \rightarrow s \gamma$ and $b \rightarrow d \gamma$ decays [8] and the isospin asymmetry between charged and neutral mesons [9]. The photon energy spectrum provides access to the distribution function of the $b$ quark inside the $B$ meson, which is a crucial input in the extraction of $\left|V_{u b}\right|$ from inclusive semileptonic $B \rightarrow X_{u} \ell \nu$ measurements [10, 11, 12, 13, 14, 15]. Finally, the decays $B \rightarrow K \pi^{+} \pi^{0} \gamma$, permit the measurement, given sufficient statistics, of the photon polarization [16]. The SM predicts nearly complete left-handed polarization.

\section{$2.1 \quad B \rightarrow K^{*} \gamma$}

The exclusive $B \rightarrow K^{*} \gamma$ modes have been studied by $B A B A R$ using a total 88 million of $B \bar{B}$ pairs [17.

We extract the final results utilizing kinematic constraints, resulting from the candidate $B$ meson reconstruction, a neural network built using event-shape variables to reduce the continuum background and a multi-dimensional extended maximum likelihood.

We find $\mathcal{B}\left(B^{0} \rightarrow K^{* 0} \gamma\right)=(3.92 \pm 0.20$ (stat) $\pm 0.24($ syst $)) \times 10^{-5}$ and $\mathcal{B}\left(B^{+} \rightarrow K^{*+} \gamma\right)=$ $(3.87 \pm 0.28($ stat $) \pm 0.26($ syst $)) \times 10^{-5}$, consistent with the SM predictions. Our measurements also constrain the direct $C P$ asymmetry to be $\mathcal{A}_{C P}=-0.013 \pm 0.036$ (stat) \pm 0.010 (syst) and the isospin asymmetry to be $\Delta_{0+}=+0.050 \pm 0.045($ stat $) \pm 0.028($ syst $) \pm 0.024\left(R^{+} / R^{0}\right)$. The asymmetries are consistent with zero and are statistically limited.

\section{$2.2 \quad B \rightarrow K \pi \pi \gamma$}

Using a total of 232 million $B \bar{B}$ pairs, $B A B A R$ has measured the partial branching fractions for $B \rightarrow K \pi \pi \gamma$ in four decay channels in the range $m_{K \pi \pi}<1.8 \mathrm{GeV}$ [18, including the $K \pi^{+} \pi^{0} \gamma$ channels important for measuring the photon polarization. Table 1 shows the signal yields and 
the computed branching fraction for all the final states. The results in the $K \pi^{+} \pi^{-}$channels are consistent with the previous measurement [19]. We present first observations of decays in the $K \pi^{+} \pi^{0}$ channels. With higher statistics it will be possible to perform a measurement of the photon polarization, but untangling the resonant contributions presents a challenge.

Table 1: Results for $B \rightarrow K \pi \pi \gamma, m_{K \pi \pi}<1.8 \mathrm{GeV}$. The first column indicates the final states, the second column shows the signal yield and the corresponding statistical error, the second column shows the branching fraction and the statistical and systematic error, respectively.

\begin{tabular}{lcc}
\hline \hline Channel & Yield & $\mathcal{B}\left(10^{-5}\right)$ \\
\hline$K^{+} \pi^{-} \pi^{+} \gamma$ & $899 \pm 38$ & $2.95 \pm 0.13 \pm 0.19$ \\
$K^{+} \pi^{-} \pi^{0} \gamma$ & $572 \pm 31$ & $4.07 \pm 0.22 \pm 0.31$ \\
$K^{0} \pi^{+} \pi^{-} \gamma$ & $176 \pm 20$ & $1.85 \pm 0.21 \pm 0.12$ \\
$K^{0} \pi^{+} \pi^{0} \gamma$ & $164 \pm 15$ & $4.56 \pm 0.42 \pm 0.30$ \\
\hline \hline
\end{tabular}

\section{$2.3 \quad$ Inclusive $b \rightarrow s \gamma$}

$B A B A R$ has recently published a paper using a sum of exclusive final states 20] to study the inclusive $b \rightarrow s \gamma$ decay. $B$ candidates are obtained combining a $s$-quark hadronic system, $X_{s}$, with a high energy photon. Kinematic constraints exploiting the fact that the $B$ is produced from the $\Upsilon(4 S)$ decay are used to suppress backgrounds. Candidates with correctly reconstructed $X_{s}$ systems are treated as signal, whereas incorrectly reconstructed systems ("cross-feed") are treated as background. This method permits a measurement of the branching fraction as a function of the hadronic mass, $M\left(X_{s}\right)$. The $M\left(X_{s}\right)$ spectrum is converted into a high resolution photon energy spectrum using the kinematic relationship for the decay of a $B$ meson of mass $M_{B}: E_{\gamma}=$ $\frac{M_{B}^{2}-M\left(X_{s}\right)^{2}}{2 M_{B}}$. where $E_{\gamma}$ is the photon energy in the $B$ rest frame which has a resolution of $1-5 \mathrm{MeV}$. The photon energy spectrum is shown in Figure 1 .

We fit the photon energy spectrum to two recent theoretical predictions, one using a combination of the operator product expansion (OPE) coupled to soft collinear effective theory (SCET) 21, 13, 14, 15, 22, 23] in the shape function mass scheme, and the other using a full OPE approach in the kinetic mass scheme [24. Figure 1] shown the best fit to the theoretical predictions, from which it can be seen that the spectrum is well described and the difference between the two schemes is small. Results are $m_{b}=4.67 \pm 0.07 \mathrm{GeV}, \mu_{\pi}^{2}=0.16_{-0.08}^{+0.10} \mathrm{GeV}^{2}$ and $m_{b}=4.70{ }_{-0.08}^{0.04} \mathrm{GeV}$, $\mu_{\pi}^{2}=0.29_{-0.04}^{+0.09} \mathrm{GeV}^{2}$, where the errors are statistical and systematic combined, for the two schemes, respectively.

Also $B A B A R$ presented preliminary results from an analysis in which the photon energy spectrum is measured without reconstructing the $X_{s}$ system, and backgrounds are suppressed using information from the rest of the event, and tagging the other $B$ of the event. Details are given in Ref. 25]. When measuring the $E_{\gamma}$ spectrum inclusively at the $\Upsilon(4 S)$ the shape of the spectrum has a large contribution from the $50 \mathrm{MeV}$ calorimeter energy resolution, and from the motion of the $B$ meson in the $\Upsilon(4 S)$ rest frame.

We calculate the first and second moments of the photon spectrum for different minimum values of the photon energy. The moments are in good agreement with predictions based on fits to the measured $b \rightarrow c \ell \nu$ moments as it can be seen in Figure 2. 


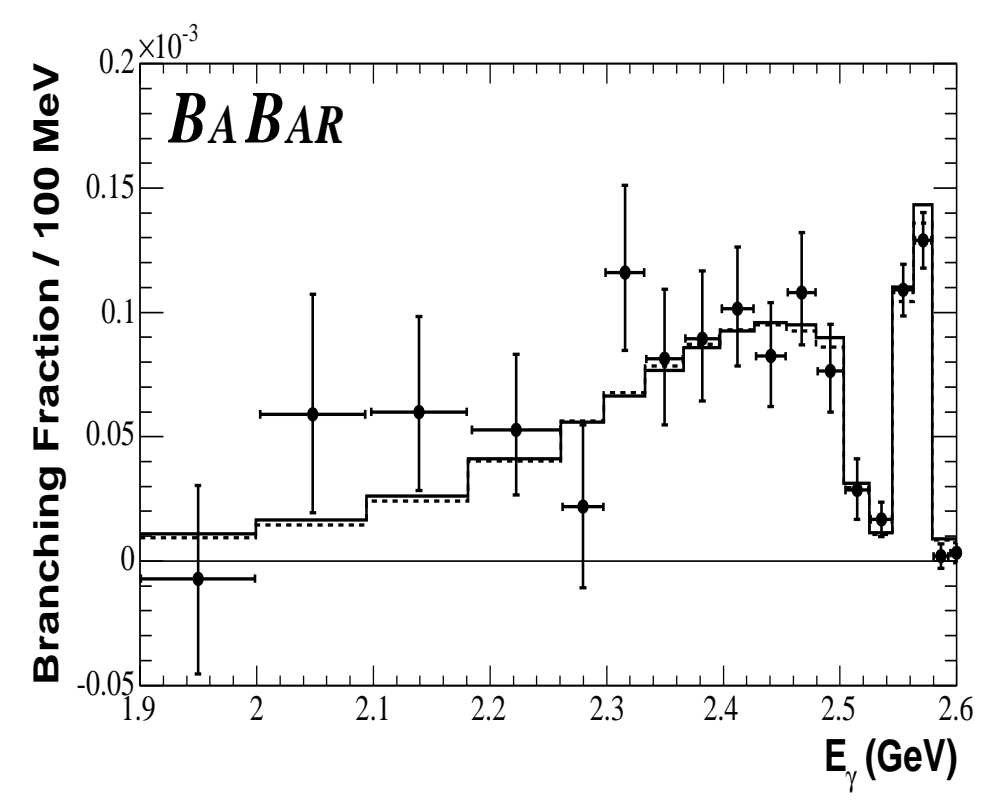

Figure 1: The photon energy spectrum according to Ref. 20. The data points are compared to theoretical predictions (histograms) obtained using the shape function (solid line) and kinetic (dashed line) schemes.
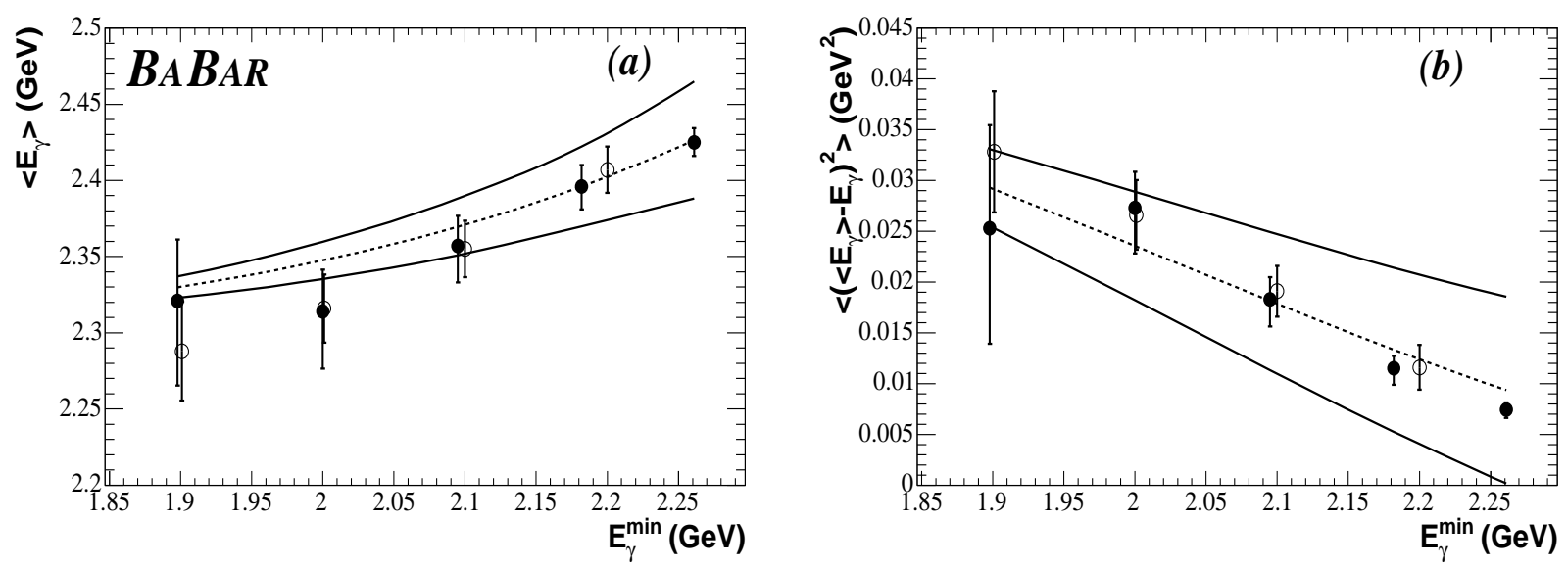

Figure 2: First (a), and second (b) moments as a function of the minimum photon energy according to Ref. [20] (solid dots) and Ref. [25] (empty dots). The dotted lines show the predicted central values based on fits to the $b \rightarrow c \ell \nu$ moments [26, and the solid lines the theoretical uncertainties from the kinetic scheme 24, 27. 
The measured branching fraction for $E_{\gamma}>1.90 \mathrm{GeV}$ are $\mathcal{B}(b \rightarrow s \gamma)=\left(3.27 \pm 0.18_{-0.40-0.09}^{+0.55+0.04}\right) \times$ $10^{-4}$ and $\mathcal{B}(b \rightarrow s \gamma)=(3.67 \pm 0.29 \pm 0.34 \pm 0.29) \times 10^{-4}$, where the errors are statistical, systematic and theoretical, respectively, for the analyses described in Refs. 20, 25]. The measured branching fractions are consistent with the SM predictions.

The direct $C P$ asymmetry between inclusive $b \rightarrow s \gamma$ and $\bar{b} \rightarrow \bar{s} \gamma$ decays, expected to be less than 0.01 in the Standard Model, is measured to be $A_{C P}(b \rightarrow s \gamma)=0.025 \pm 0.050($ stat $) \pm 0.015$ (syst) [28], which is statistically limited and consistent with the SM predictions. Looking at both $s$ and $d$ final states, the measured $C P$ asymmetry is $A_{C P}(b \rightarrow(s+d) \gamma)=-0.110 \pm 0.115($ stat $) \pm 0.017$ (syst) [25], still statistically limited.

Finally, using the semi-inclusive method, we have made the first measurement of the isospin asymmetry between $B^{-} \rightarrow X_{s \bar{u}} \gamma$ and $\bar{B}^{0} \rightarrow X_{s \bar{d}} \gamma$. We obtain $\Delta_{0-}=-0.006 \pm 0.058 \pm 0.009 \pm 0.024$ which is consistent with zero within the experimental uncertainty, which is mainly statistical.

\section{$3 \quad b \rightarrow d \gamma$ final states}

Studies of the $b \rightarrow d \gamma$ decays for now focus primarily on searching for the exclusive process $B \rightarrow$ $\rho / \omega \gamma$. Both inclusive and exclusive $b \rightarrow d \gamma$ decays are suppressed by $\left|V_{t d} / V_{t s}\right|^{2} \sim 0.04$ with respect to the corresponding $b \rightarrow s \gamma$ modes. The branching fraction is predicted to be in the range $\mathcal{B}(B \rightarrow \rho \gamma)=(0.9-2.7) \times 10^{-6}[29$, while the $C P$ asymmetry is of the order of $10 \%$ [29].

From the experimental point of view, $B \rightarrow \rho(\omega) \gamma$ is more difficult than $B \rightarrow K^{*} \gamma$ because the backgrounds are bigger since this mode is CKM suppressed and $u \bar{u}, d \bar{d}$ continuum processes are enhanced compared to $s \bar{s}$ continuum processes.

The upper limits at 90\% CL on the exclusive decays $B \rightarrow \rho(\omega) \gamma$ from BABAR [30], which uses a neural network to suppress most of the continuum background and particle identification to veto kaons, are $0.4,1.8$ and $1.0 \times 10^{-6}$ on $\rho^{0} \gamma, \rho^{+} \gamma$ and $\omega \gamma$, respectively, using 211 million $B \bar{B}$ events. Assuming isospin symmetry, this gives a combined limit $\mathcal{B}(B \rightarrow \rho \gamma)<1.2 \times 10^{-6}$ (90\% C.L.). Using 386 million $B \bar{B}$ decays, Belle observed a signal and measured a combined limit $\mathcal{B}(B \rightarrow \rho \gamma)=\left(1.34_{-0.31}^{+0.34}(\text { stat })_{-0.10}^{+0.14}(\right.$ syst $\left.)\right) \times 10^{-6}$ [31], in the region already excluded by BABAR at $90 \%$ C.L. Updating the measurements using a larger statistical sample will help in understanding the difference among the results obtained by the two Collaborations.

Of particular theoretical interested is the ratio $\mathcal{B}(B \rightarrow \rho \gamma)$ to $\left(B \rightarrow K^{*} \gamma\right)$ as most of the theoretical uncertainty cancels and can be used to determine the ratio $\left|V_{t d} / V_{t s}\right|$. Combining the results from $B A B A R$ and Belle assuming isospin asymmetry, a branching fraction of $\mathcal{B}(B \rightarrow$ $\rho \gamma)=\left(0.94_{+0.25}^{-0.22}\right) \times 10^{-6}$ is measured, from which a constraint on the CKM elements $\left|V_{t d} / V_{t s}\right|$ of $0.18 \pm 0.03$ is obtained (see Ref. [32] for details).

\section{$4 \quad B^{0} \rightarrow D^{* 0} \gamma$}

Within the Standard Model, the rare decay $B^{0} \rightarrow D^{* 0} \gamma$ is dominated by the $W$-boson exchange process. Similar $W$-exchange transitions are present in other decays. For example, they contribute to the decay $B^{0} \rightarrow \rho^{0} \gamma$ along with the leading electromagnetic-penguin process. The presence of an annihilation contribution in $B \rightarrow \rho \gamma$ which is not present in $B \rightarrow K^{*} \gamma$ decays and which is associated to a different CKM factor $\left(\sim\left|V_{u b}^{*} V_{u d}\right|\right)$ represents a theoretical error in the extraction of $\left|V_{t d} / V_{t s}\right|$.

Using 88 million $B \bar{B}$ pairs, $B A B A R$ sets an upper limit on the branching fraction of $\mathcal{B}\left(B^{0} \rightarrow\right.$ $\left.D^{* 0} \gamma\right)<2.5 \times 10^{-5}$ at the $90 \%$ confidence level [33], twice as stringent as the previous limit. 


\section{$5 \quad b \rightarrow s \ell^{+} \ell^{-}$final states}

The rare decay $B \rightarrow X_{s} \ell^{+} \ell^{-}$, which proceeds through the $b \rightarrow s \ell^{+} \ell^{-}$transition, is forbidden at lowest order in the SM but is allowed at higher order via electroweak penguin and $W$-box diagrams. New Physics can appear in the loops and change the rate [34, 35, the asymmetry [37] and the kinematic distributions.

$B A B A R$ has measured the branching fractions and direct $C P$ asymmetries $A_{C P}$ of the rare decays $B \rightarrow K \ell^{+} \ell^{-}$and $B \rightarrow K^{*} \ell^{+} \ell^{-}$, using a total of 229 million $B \bar{B}$ pairs [38].

We select events that include two oppositely charged electrons or muons, a kaon candidate and, for the $B \rightarrow K^{*} \ell^{+} \ell^{-}$modes, a $\pi^{ \pm}$candidate that, when combined with a kaon candidate, forms a $K^{*}$ candidate. Combinatorial background from continuum processes is suppressed using a Fisher discriminant. Background $B$ decays to charmonium are suppressed excluding dilepton pairs consistent with the $J / \psi$ and $\psi(2 S)$ masses. Fits to the kinematic variables of the reconstructed $B$ mesons are performed to extract the signal events.

We find the (lepton-flavor-averaged, $B$-charge-averaged) branching fractions $\mathcal{B}\left(B \rightarrow K \ell^{+} \ell^{-}\right)=$ $(0.34 \pm 0.07 \pm 0.03) \times 10^{-6}$ and $\mathcal{B}\left(B \rightarrow K^{*} \ell^{+} \ell^{-}\right)=\left(0.78_{-0.17}^{+0.19} \pm 0.12\right) \times 10^{-6}$, consistent with the $\mathrm{SM}$ predictions for these modes. We measure the direct $C P$ asymmetries $A_{C P}\left(B^{+} \rightarrow K^{+} \ell^{+} \ell^{-}\right)=$ $0.08 \pm 0.22 \pm 0.11$ and $A_{C P}\left(B \rightarrow K^{*} \ell^{+} \ell^{-}\right)=-0.03 \pm 0.23 \pm 0.12$ where the systematic uncertainty is dominated by the unknown asymmetry in the peaking backgrounds.

Studying separately electron and muon final states, we find the ratio of muon to electron branching fractions over the full range of $q^{2}$ to be $R_{K}=1.06 \pm 0.48 \pm 0.05$ and $R_{K^{*}}=0.93 \pm 0.46 \pm 0.06$ where these are expected in the Standard Model to be 1.00 and 0.75 , respectively, with small theoretical uncertainties.

All measurements are consistent with the SM predictions and are statistically limited.

$B A B A R$ also observed the inclusive decay $B \rightarrow X_{s} \ell \ell$. Details can be found in Ref. 39].

\section{$6 \quad B^{+} \rightarrow \tau^{+} \nu_{\tau}$}

We search for the rare leptonic decay $B^{+} \rightarrow \tau^{+} \nu_{\tau}$ in a sample of $232 \times 10^{6} B \bar{B}$ pairs [40]. This decay is helicity favoured with respect to the corresponding decays with electrons and muons. Signal events are selected by examining the properties of the $B$ meson recoiling against the semileptonic decay $B^{-} \rightarrow D^{* 0} \ell^{-} \bar{\nu}_{\ell}$. We find no evidence for a signal and set an upper limit on the branching fraction of $\mathcal{B}\left(B^{+} \rightarrow \tau^{+} \nu_{\tau}\right)<2.8 \times 10^{-4}$ at the $90 \%$ confidence level. We combine this result with a previous, statistically independent $B A B A R$ search for $B^{+} \rightarrow \tau^{+} \nu_{\tau}$ to give an upper limit of $\mathcal{B}\left(B^{+} \rightarrow \tau^{+} \nu_{\tau}\right)<2.6 \times 10^{-4}$ at the $90 \%$ confidence level. These result represents the most stringent limit on $B^{+} \rightarrow \tau^{+} \nu_{\tau}$ reported to date. Using limits on $B \rightarrow \tau \nu$ from both BABAR and Belle, a value $f_{B}=0.178 \pm 0.062 \mathrm{GeV}$ is obtained from fits to the unitarity triangle (see Ref. 32. for details).

\section{Conclusions}

A review of recent experimental results on electro-weak and leptonic decays is presented. In particular, we have focussed on the new results on the $b \rightarrow s(d) \gamma, B \rightarrow D^{* 0} \gamma, b \rightarrow$ sll and $B \rightarrow \tau \nu$ decays. 


\section{Acknowledgements}

We are grateful to Steve Playfer for several useful discussions. We would like to thank the organizers of BEAUTY 2005 for the invitation and for having organized a very interesting conference.

\section{References}

[1] BABAR collaboration, B. Aubert et al., Nucl. Instrum. Methods A 479, 1 (2002).

[2] B. Grinstein and M.B. Wise, Phys. Lett. B 201, 274 (1988).

[3] W.S. Hou and R.S. Willey, Phys. Lett. B 202, 591 (1988).

[4] J.L. Hewett and J.D. Wells, Phys. Rev. D 55, 5549 (1997).

[5] A.L. Kagan and M. Neubert, Eur. Phys. J. C 7, 5 (1999).

[6] T. Hurth, Rev. Mod. Phys. 75, 1159 (2003).

[7] A.L. Kagan and M. Neubert, Phys. Rev. D 58, 094012 (1998).

[8] T. Hurth, E. Lunghi and W. Porod, Nucl. Phys. B 704, 56 (2005.)

[9] A.L. Kagan and M. Neubert, Phys. Lett. B 539, 227 (2002).

[10] I.I. Bigi and N. Uraltsev, Int.J.Mod.Phys.A17, 4709 (2002).

[11] I.I. Bigi and N. Uraltsev, Phys. Lett. B 579, 340 (2004).

[12] C.W. Bauer and A.V. Manohar, Phys. Rev. D 70, 034024 (2004).

[13] S. W. Bosch, B. O. Lange, M. Neubert and G. Paz, Nucl. Phys. B 699, 335 (2004).

[14] S.W. Bosch, M. Neubert and G. Paz, JHEP 0411, 073 (2004).

[15] B.O. Lange, M. Neubert and G. Paz, hep-ph/0504071, accepted by Phys. Rev. D.

[16] M. Gronau, et al., Phys. Rev. Lett. 88, 051802 ((2002);)M. Gronau and D. Pirjol, Phys. Rev. D 66, 054008 (2002).

[17] BABAR collaboration, B. Aubert et al., Phys. Rev. D 70, 112006 (2004).

[18] BABAR collaboration, B. Aubert et al., hep-ex/0507031, presented at the International Europhysics Conference On High Energy Physics (HEP 2005).

[19] Belle Collaboration, H. Yang et al., Phys. Rev. Lett. 94,, 111802 ((2005).)

[20] BABAR collaboration, B. Aubert et al., hep-ex/0508004, accepted by Phys. Rev. D.

[21] M. Neubert, Eur. Phys. J. C 40, 165 (2005).

[22] M. Neubert, hep-ph/0411027, submitted to Eur. Phys. J. C.

[23] M. Neubert, Phys. Lett. B 612, 13 (2005). 
[24] D. Benson, I.I. Bigi and N. Uraltsev, Nucl. Phys. B 710, 371 (2005).

[25] BABAR collaboration, B. Aubert et al., hep-ex/0507001, contributed to the XXIInd International Symposium on Lepton and Photon Interactions at High energies (LP 2005).

[26] BABAR collaboration, B. Aubert et al., Phys. Rev. Lett. 93, 011803 (2004).

[27] D. Benson, I.I. Bigi and N. Uraltsev, private communication.

[28] BABAR collaboration, B. Aubert et al., Phys. Rev. Lett. 93, 021804 (2004).

[29] T. Huang Z Li, H. Zhang, J. Phys. G25, 1179 (1999) ; A. Ali and A.Y. Parkhomenko Eur. Phys. J. C 23, 89 (2003); S. Bosch and G. Buchalla, Nucl. Phys. B 621, 459 (2002); R. Fleischer and S. Recksiegel Phys. Rev. D 71, 051501 (2005).

[30] BABAR Collaboration, B. Aubert et al., Phys. Rev. Lett. 94, 011801 (2005).

[31] Belle collaboration, K. Abe et al., Belle Collaboration, hep-ex/0506079

[32] M. Bona et al. [UTfit Coll.] "The 2004 UTfit Collaboration Report on the Status of the Unitarity Triangle in the Standard Model", hep-ph/0501199 and updates on the web site http://utfit.roma1.infn.it/.

[33] BABAR collaboration, B. Aubert et al., hep-ex/0506070, accepted by Phys. Rev. D (Rapid Communications).

[34] A. Ali et al., Phys. Rev. D 66, 034002 (2002).

[35] T. Hurth, Rev. Mod. Phys. 75, 1159 (2003).

[36] F. Krüger, L. M. Sehgal, N. Sinha, and R. Sinha, Phys. Rev. D 61, 114028 (2000); Erratumibid. D 63, 019901 (2001).

[37] F. Krüger and E. Lunghi,Phys. Rev. D 63, 014013 (2001).

[38] BABAR Collaboration, B. Aubert et al., hep-ex/0507005, contributed to the XXIInd International Symposium on Lepton and Photon Interactions at High energies (LP 2005).

[39] BABAR Collaboration, B. Aubert et al., Phys. Rev. Lett. 93, 081802 (2004).

[40] BABAR Collaboration, B. Aubert et al., hep-ex/0507069, submitted to Phys. Lett. B. 